\title{
Health-related Quality of life in 640 head and neck cancer survivors after radiotherapy using EORTC QLQ-C30 and QLQ-H\&N35 questionnaires
}

\author{
Stephen Wan Leung ${ }^{1,2 \dagger}$, Tsair-Fwu Lee ${ }^{3 \dagger}$, Chih-Yen Chien ${ }^{4}$, Pei-Ju Chao ${ }^{3,5}$, Wen-Ling Tsai ${ }^{6}$ and Fu-Min Fang ${ }^{5^{*}}$
}

\begin{abstract}
Background: With the advances in modern radiotherapy (RT), many patients with head and neck cancer (HNC) can be effectively cured, and their health-related quality of life (HR-QoL) has become an important issue. In this study, we evaluated the prognosticators of HR-QoL in a large cohort of HNC patients, with a focus on the result from technological advances in RT.

Methods: A cross-sectional investigation was conducted to assess the HR-QoL of $640 \mathrm{HNC}$ patients with cancerfree survival of more than 2 years. Among them, 371 patients were treated by two-dimensional RT (2DRT), 127 by three-dimensional conformal RT (3DCRT), and 142 by intensity-modulated RT (IMRT). The EORTC QLQ-C30 questionnaire and QLQ-H\&N35 module were used. A general linear model multivariate analysis of variance was used to analyze the prognosticators of HR-QoL.

Results: By multivariate analysis, the variables of gender, annual family income, tumor site, AJCC stage, treatment methods, and RT technique were prognosticators for QLQ-C30 results, so were tumor site and RT technique for H\&N35. Significant difference $(p<0.05)$ of HR-QoL outcome by different RT techniques was observed at 2 of the 15 scales in QLQ-C30 and 10 of the 13 scales in H\&N35. Compared with 2DRT, IMRT had significant better outcome in the scales of global QoL, physical functioning, swallowing, senses (taste/smell), speech, social eating, social contact, teeth, opening mouth, dry mouth, sticky saliva, and feeling ill.

Conclusions: The technological advance of RT substantially improves the head-and-neck related symptoms and broad aspects of HR-QoL for HNC survivors.
\end{abstract}

\section{Background}

Health-related quality of life (HR-QoL) and its assessment have become increasingly important in health care, especially in the field of chronic diseases. Conventionally, the endpoints of medical care for cancer patients usually focused on the so-called survival rate, local control rate, or complication rate. These endpoints were usually assessed from the physician's points of view. These assessments lacked knowledge and understanding of the patients' mental and emotional well being. HR-QoL generally refers to the patient's perception of the effects of the disease and the impact on the

\footnotetext{
* Correspondence: fang2569@adm.cgmh.org.tw

+ Contributed equally

${ }^{5}$ Radiation Oncology, Chang Gung Memorial Hospital - Kaohsiung Medical

Center, Chang Gung University College of Medicine, Kaohsiung, Taiwan
}

Full list of author information is available at the end of the article patient's daily functioning, and has two fundamental premises. First, it is a multi-dimensional survey incorporating physical, psychological, social, and emotional functional domains. Second, it is subjective and must be self-reporting, according to the patient's own experiences [1].

Determining how to measure and quantify the subjective experience of HR-QoL has been a challenging issue. There are now a variety of well-validated HR-QoL instruments available for use in the field of oncology. Three types of methods have been categorized. They include the generic type, e.g. the Short Form-36 (SF-36), the cancer specific type, e.g. the Functional Assessment of Cancer Treatment (FACT-G), the European Organization of Research and Treatment of Cancer Quality of Life Core Questionnaire, version 3.0 (EORTC QLQ$\mathrm{C} 30$ ), and the cancer site-specific type, e.g. the head and

\section{Ciomed Central}


neck modules in EORTC (EORTC QLQ-HN35), and FACT (FACT-HN) [2-6].

Perhaps in no other group of cancer patients does HR-QoL present as important a role as in HNC patients. This is because they may have obviously debilitating problems with swallowing, speech, and hearing, as well as the psychological effects of loss of function and change in body image [7]. Radiotherapy is one of the most important treatment modalities for HNC patients, either in a definite way or a combination with surgery and/or chemotherapy $(\mathrm{C} / \mathrm{T})$. Over the past decade, the advances of RT techniques for treating HNC have emerged from so-called two-dimensional RT (2DRT) to the three-dimensional conformal RT (3DCRT) and intensity-modulated RT (IMRT). 2DRT has proven effective in the treatment of HNC. However, complications associated with irradiation of sensitive normal structures, such as the salivary glands in the path of the irradiation, are still remarkable and often lifelong. The reliance of 3DCRT and IMRT on computed tomography-guided 3D planning allows better delineation of tumor target and organs at risk with clearer radiological visualization of their spatial relations, thus providing a potentially therapeutic benefit of dose escalation to tumor tissue with reduced toxicity to normal tissues [8].

IMRT represents an advanced form of 3DCRT. It employs inverse planning algorithms and iterative computer-driven optimization to generate treatment fields with varying beam intensity. Combinations of intensitymodulated fields produce custom-tailored conformal dose distributions around the tumor, with steep dose gradients at the transition to adjacent normal tissues. Growing reports have shown that the technical and dosimetric superiority of 3DCRT and IMRT over 2DRT can translate into clinical benefits, such as reduced normal tissue toxicity (e.g., parotid gland sparing), improved local control, or even patient survival [9-14].

Radical RT for treating HNC was routinely delivered by 2DRT in our hospital before the introduction of 3DCRT in April 1996. From April 1996 to March 2002, 3DCRT was gradually used to replace 2DRT. After becoming familiar with the techniques of 3DCRT and implementation of the IMRT system by March 2002, the physicians and physicists in our institute began to use the two techniques in treating HNC. In a previous publication, we have reported that HNC survivors had significantly poorer HR-QoL outcomes compared with Taiwanese norms $[15,16]$. In this study, we further compared the HR-QoL results assessed by the EORTC QLQ-C30 and QLQ-H\&N35 modules for HNC survivors who, as a result of technological advances in $\mathrm{RT}$ at our institute, were treated with 2DRT, 3DCRT, or IMRT in different time periods.

\section{Methods}

\section{Study population}

This study is a cross sectional investigation, analyzing HR-QoL data of HNC patients who were cancer free when their HR-QoL was assessed during the period from January 2005 to December 2008. Eligibility criteria of patients included 1) pathologically proven $\mathrm{HNC}$ at nasopharynx, oral cavity, oropharynx, hypopharynx, or larynx, 2) receiving $\mathrm{RT}$ and regular follow-up at the department of radiation oncology at Chang Gung Memorial Hospital - Kaohsiung Medical Center, 3) cancer free survival more than two years after RT, and 4) completion of the self-reported questionnaire. Six hundred and forty HNC patients, treated with definite or postoperative RT, were collected and informed consent was obtained from all of them. They included 371 patients treated by $2 \mathrm{DRT}$ and 269 patients by conformal RT (3DCRT: 127 patients, IMRT: 142 patients). Concerning the existence of selection bias, we compared the distributions of sociodemographic characteristics (including age, gender, marital status, and education level) between HNC survivors in the study and all other surviving HNC patients $(n=221)$ found in the cancer registry database in the department. No statistically significant differences were found between them (data not shown).

Patient characteristics including sociodemographic variables and cancer- or treatment- related variables are listed in Table 1. The cancer stages were according to the staging system of the American Joint Committee on Cancer (AJCC $6^{\text {th }}$ edition) published in 2002. The comorbidity status was recorded according to the Charlson comorbidity index by review of chart and on the basis of self-report [17]. A summary of the primary cancer site included 316 cases (49\%) of nasopharyx, 129 (20\%) of oral cavity, 75 (12\%) of oropharynx, 75 (12\%) of hypopharynx, and 45 (7\%) of larynx. As regards the sociodemographic information, $86 \%$ of them were educated $\leqq 12$ years, $53 \%$ with an annual family income $\leqq 1.2$ million NTD (1USD = 33NTD), 65\% not employed, and $19 \%$ without a spouse (unmarried or divorced). Four hundred and thirty patients (67\%) had AJCC stage III or IV disease and $267(42 \%)$ patients had at least one kind of comorbidity. The major treatment was surgery in 249 (39\%) patients and RT alone or plus C/T in 391 (61\%) patients. Uneven distribution existed between the 2DRT and 3DCRT/IMRT group. The 3DCRT/IMRT group had a higher distribution in patients with lower annual family income, non-nasopharyngeal cancer site, stage III-IV, surgery, chemotherapy, or shorter survival years. The median (range) follow-up years of patients after treatment when their HR-QoL data were collected were 5.2 (2.8-14.1), 3.9 (2.1-10.3), and 3.1 (2.0-6.5) years in the 2DRT, 3DCRT, and IMRT group, respectively. This 
Table 1 Patient characteristics $(n=640)$

\begin{tabular}{|c|c|c|c|c|}
\hline Variables & Total & 2DRT & 3DCRT & IMRT \\
\hline Patient number & 640 & 371 & 127 & 142 \\
\hline Age, median (range) years & $\begin{array}{l}52 \\
(15-87)\end{array}$ & $\begin{array}{l}52 \\
(15-87)\end{array}$ & $\begin{array}{l}53 \\
(31-83)\end{array}$ & $\begin{array}{l}51 \\
(23-79)\end{array}$ \\
\hline Male/female & $537 / 103$ & $297 / 74$ & $117 / 10$ & $123 / 19$ \\
\hline \multicolumn{5}{|l|}{ Education years } \\
\hline$\leqq 6$ & 245 & 147 & 52 & 46 \\
\hline $6 \sim 12$ & 305 & 168 & 64 & 73 \\
\hline$>12$ & 90 & 56 & 11 & 23 \\
\hline \multicolumn{5}{|l|}{$\begin{array}{l}\text { Annual family income, (104 } \\
\text { NTD) }\end{array}$} \\
\hline$<60$ & 119 & 53 & 30 & 36 \\
\hline $60 \sim 120$ & 218 & 125 & 47 & 46 \\
\hline$\geq 120$ & 303 & 193 & 50 & 60 \\
\hline \multicolumn{5}{|l|}{ Marital status } \\
\hline With spouse & 518 & 298 & 102 & 118 \\
\hline Without spouse & 122 & 73 & 25 & 24 \\
\hline \multicolumn{5}{|l|}{ Employment } \\
\hline Yes & 225 & 139 & 36 & 50 \\
\hline No & 415 & 232 & 91 & 92 \\
\hline \multicolumn{5}{|l|}{ Cancer sites } \\
\hline Nasopharynx & 316 & 226 & 31 & 59 \\
\hline Oral cavity & 129 & 64 & 33 & 32 \\
\hline Oropharynx & 75 & 41 & 21 & 13 \\
\hline Hypoppharynx & 75 & 20 & 28 & 27 \\
\hline Larynx & 45 & 20 & 14 & 11 \\
\hline \multicolumn{5}{|l|}{ AJCC stage } \\
\hline$|-| \mid$ & 210 & 145 & 26 & 39 \\
\hline III-IV & 430 & 226 & 101 & 103 \\
\hline \multicolumn{5}{|l|}{ Radiation dose } \\
\hline$\leqq 70.2 \mathrm{~Gy}$ & 281 & 126 & 81 & 74 \\
\hline$>70.2 \mathrm{~Gy}$ & 359 & 245 & 46 & 68 \\
\hline \multicolumn{5}{|l|}{ Surgery } \\
\hline No & 391 & 264 & 53 & 74 \\
\hline Yes & 249 & 107 & 74 & 68 \\
\hline \multicolumn{5}{|l|}{ Chemotherapy } \\
\hline No & 343 & 237 & 57 & 49 \\
\hline Yes & 297 & 134 & 70 & 93 \\
\hline \multicolumn{5}{|l|}{ Comorbidity score } \\
\hline 0 & 373 & 219 & 66 & 88 \\
\hline$\geqq 1$ & 267 & 152 & 61 & 54 \\
\hline Follow-up years, median & 4.3 & 5.2 & 3.9 & 3.1 \\
\hline
\end{tabular}

Abbreviations: $\mathrm{RT}=$ radiotherapy; $2 \mathrm{DRT}=$ two dimensional $\mathrm{RT} ; 3 \mathrm{DCRT}=$ three dimensional conformal RT; IMRT = intensity-modulated RT; 1USD = 33NTD;

AJCC = American Joint Committee on Cancer, 6th edition; Comorbidity score was based on Charlson comorbidity index.

study was approved by the appropriate institutional review boards (IRB) of the hospital.

\section{Techniques of RT}

\section{DRT}

The detailed portal arrangement and dosing of conventional 2DRT in HNC have been described previously
$[18,19]$. Briefly, 2DRT was given in two phases, namely before and after 44 46.8 Gy of the spinal cord tolerance dose. In the first phase, patients were irradiated by a 6 MV photon beam with a daily fraction of 1.8 or $2.0 \mathrm{~Gy}$ (5 6 fractions per week) via bilateral opposing faciocervical fields and one lower anterior cervical field. For definite RT, the target covers the primary tumor with surrounding anatomic area and regional neck lymphatics. For postoperative cases, the surgical tumor and nodal bed as well as the prophylactic risky nodal area were included. In the second phase, the gross tumor was boosted to $64.8 \sim 81 \mathrm{~Gy}$ in definite RT and tumor bed to 57.6 64.8 Gy in postoperative cases via bilateral opposing photon beams to shield the spinal cord. Residual neck lymph nodes or risky nodal bed area were simultaneously boosted by a 9- or $12-\mathrm{Mev}$ electron beam to 56 79 Gy, depending on the nodal situation.

\section{DCRT}

The immobilization, treatment targets, and dose/fractionation prescription of 3DCRT in treating $\mathrm{HNC}$ in our institute primarily followed the guidelines for 2DRT. The Cadplan (Varian, Milpitas, CA) or Pinnacle 3D treatment planning system (Pinnacle3, Philips, Fitchburg, WI) was used. The technical details of 3DCRT in HNC have been addressed [19,20]. For each patient, 5 or 7 coplanar portals were usually designed. Shrinkage of the clinical target volume (CTV) volume was usually performed after the tumoricidal dose of 45.0-50.4 Gy was reached for the microscopic lesions. The 90-95\% isodose volume to cover the planning target volume (PTV) with the spinal cord strictly limited below the $60 \%$ isodose line was applied.

\section{IMRT}

The immobilization, target definition and delineation, and dose/fractionation prescription of IMRT for HNSCC treated by combined modality were approximately the same as described above for 3DCRT. We used the Cadplan or Pinnacle treatment planning system to perform the inverse planning and dose optimisation. For each patient, IMRT plans with five or seven coplanar portals were created. The delivery of the plans was performed in Varian machines equipped with dynamic multi-leaf collimators. The dose/fractionation prescription of IMRT primarily followed the guidelines for 3DRT [16,21].

\section{Instruments of HR-QoL}

The Taiwan Chinese versions of the EORTC QLQ-C30 and QLQ-H\&N35 questionnaires were obtained from the Quality of Life Unit, EORTC Data Center in Brussels, Belgium [4,6,22]. The EORTC questionnaires were chosen for this research because it is one of the most widely implemented questionnaires, with over 10 years of research invested to develop an integrated, modular 
approach, as well as utilization of the instrument in international clinical trials, and the Taiwan Chinese version is available and easily completed by our patients [23]. The EORTC QLQ-C30 incorporates a range of HR-QoL issues relevant to a broad range of cancer patients. It has been translated into many languages and validated for many types of cancer, including head-andneck cancer. It contains five functional scales (physical, role, cognitive, emotional and social), three symptom scales (fatigue, pain, and nausea/vomiting), a global QoL scale, and six single-items (dyspnea, insomnia, appetite loss, constipation, diarrhea, and financial difficulties). The QLQ-H\&N35 is a module used for assessing the HR-QoL for head-and-neck cancer patients. It incorporates seven multiple-item scales that assess the symptoms of pain, swallowing ability, senses (taste/smell), speech, social eating, social contact, and sexuality. Also included are six single-item scales, which survey the presence of symptomatic problems associated with teeth, mouth-opening, dry mouth (xerostomia), sticky saliva, coughing, and feeling ill. All scales pertaining to the EORTC QLQ-C30 and QLQ-H\&N35 range from zero to 100. A high score for a functional or global HR-QoL scale represents a relatively high/healthy level of functioning or global quality of life, whereas a high score for a symptom scale represents the presence of a symptom or $\operatorname{problem}(\mathrm{s})$.

\section{Statistic analysis}

The mean scores and standard deviations of the HRQoL scales were calculated according to the EORTC QLQ scoring manual [24]. To analyze the correlations between the factors and the HR-QoL scales, general linear model (GLM) multivariate analysis of variance (MANOVA) was used [25]. The GLM-MANOVA approach is used to test the hypothesis of a significant association between a set of interrelated dependent variables (HR-QoL scales) and independent variables. The independent variables analyzed in the present study consisted of five sociodemographic variables: age $(<40 v$. $40 \sim 60 v$. $\geq 60$ years), sex (male $v$. female), years of education years ( $\leq 6 \nu .6 \sim 12 v .>12$ years), marital status (with $v$. without spouse), and annual family income $(<0.6 v .0 .6 \sim 1.2 v . \geq 1.2$ million NTD), and six clinical factors: CCI score $\left(0 v_{.} \geq 1\right)$, tumour site (Oral cavity $v$. Oropharynx $v$. Hypopharynx/Larynx $v$. Nasopharynx), AJCC stage (stage I-II $v$. III-IV), treatment methods (surgery + RT $v$. surgery $+\mathrm{RT}+\mathrm{C} / \mathrm{T} v$. RT $\pm \mathrm{C} / \mathrm{T}$ ), RT technique (2DRT $v$. 3DCRT $v$. IMRT), and follow-up years $(2 \sim 3 v .3 \sim 5 v$. > 5).

GLM-MANOVA was performed for QLQ-C30 and H\&N35 separately and in the following two steps. First, to investigate the association of a given factor with HRQoL scales, a univariate analysis was conducted to establish whether the factor was associated significantly with any of the HR-QoL scales. Wilks' $\lambda$ was used to test the impact of each variable included in the model. All variables were entered into the multi-factor model. In case of a significant association between a factor and all HR-QoL scales taken together, a second ANOVA was performed to investigate the association between that prognostic factor and each HR-QoL scale separately, with post-hoc testing using the Bonferroni method with a $p$-value $<0.05$ from the two-sided test regarded to be statistically significant.

Being the most concerned scales of HR-QoL in the study, the global QoL and xerostomia were further analyzed by multiple linear regression models to explore their associated prognosticators, respectively. All the data processing was performed using the statistic software SPSS for Windows (version 15.0; SPSS Inc., Chicago, IL).

\section{Results}

\section{Outcomes of HR-QoL}

The mean score for global QoL was 54.6. The value of the five scales of functioning ranged from 75.1 (social functioning) to 86.4 (role functioning). The highest symptom score on QLQ-C30 was for fatigue, followed by financial problems and insomnia. In the H\&N35 module, dry mouth, sticky saliva, and tooth problems ranked as the three worst symptoms. (Table 2)

\section{Variables associated with HR-QoL outcomes}

In the first step of the GLM-MANOVA, the association between the independent variables (five sociodemographic and six clinical variables) and the dependent variables (15 scales of QLQ-C30) was investigated (onefactor model, Table 3 ). This analysis showed that 10 of the 11 variables (except the length of follow-up) ( $p<$ $0.05)$ were associated with the overall outcome on QLQ-C30. The 11 variables were then entered into the multifactor model analysis, which indicated that the variables of gender, annual family income, tumor site, AJCC stage, treatment methods, and RT technique remained significant. The same statistical procedures were repeated for the analysis of the association between the 11 independent variables and the scales of H\&N35. In a one-factor model, four of the five sociodemographic variables and four of the six clinical variables (except $\mathrm{CCI}$ and the length of follow-up) were significantly ( $p<$ 0.05 ) associated with the overall outcome on H\&N35. In the multifactor model, only tumor site and RT technique remained significant (Table 3 ).

The variables specifically associated with global QoL and xerostomia were further analyzed. As demonstrated by linear regression model in Table 4 we observed a significant trend that survivors with $\mathrm{CCI} \geq 1(\beta=5.2, \mathrm{p}=$ 
Table 2 Calculated scores of EORTC QLQ-C30 and H\&N35 scales for head and neck cancer survivors

\begin{tabular}{|c|c|c|c|}
\hline$\underline{\text { Scales }}$ & Mean (SD) & Median & Range \\
\hline \multicolumn{4}{|l|}{ QLQ-C30 } \\
\hline Global quality of life & $54.6(19.9)$ & 50.0 & $0.0-100.0$ \\
\hline Physical functioning & $84.5(16.5)$ & 86.7 & $11.1-100.0$ \\
\hline Role functioning & $86.4(21.0)$ & 100.0 & $0.0-100.0$ \\
\hline Emotional functioning & $77.9(20.0)$ & 75.0 & $0.0-100.0$ \\
\hline Cognitive functioning & $78.4(19.8)$ & 83.3 & $0.0-100.0$ \\
\hline Social functioning & $75.1(24.2)$ & 66.7 & $0.0-100.0$ \\
\hline Fatigue & $29.2(20.4)$ & 33.3 & $0.0-100.0$ \\
\hline Nausea/Nomiting & $9.3(17.5)$ & 0.0 & $0.0-100.0$ \\
\hline Pain & $21.9(22.4)$ & 16.7 & $0.0-100.0$ \\
\hline Dyspnea & $15.2(21.6)$ & 0.0 & $0.0-100.0$ \\
\hline Insomnia & $26.3(25.9)$ & 33.3 & $0.0-100.0$ \\
\hline Appetite loss & $19.9(24.6)$ & 0.0 & $0.0-100.0$ \\
\hline Constipation & $19.1(23.7)$ & 33.3 & $0.0-100.0$ \\
\hline Diarrhea & $14.0(19.9)$ & 0.0 & $0.0-100.0$ \\
\hline Financial problems & $26.4(27.1)$ & 33.3 & $0.0-100.0$ \\
\hline \multicolumn{4}{|l|}{ H\&N35 } \\
\hline Pain & 19.1 (20.6) & 16.7 & $0.0-100.0$ \\
\hline Swallowing & $30.2(24.4)$ & 25.0 & $0.0-100.0$ \\
\hline Senses (taste/smell) & $26.9(27.7)$ & 25.0 & $0.0-100.0$ \\
\hline Speech & $28.0(25.9)$ & 22.2 & $0.0-100.0$ \\
\hline Social eating & $28.5(26.9)$ & 25.0 & $0.0-100.0$ \\
\hline Social contact & $20.4(23.5)$ & 13.3 & $0.0-100.0$ \\
\hline Sexuality & $26.0(27.7)$ & 33.3 & $0.0-100.0$ \\
\hline Teeth & $38.9(29.8)$ & 33.3 & $0.0-100.0$ \\
\hline Opening mouth & 32.8 (31.6) & 33.3 & $0.0-100.0$ \\
\hline Dry mouth & $48.3(31.0)$ & 33.3 & $0.0-100.0$ \\
\hline Sticky saliva & 40.7 (30.6) & 33.3 & $0.0-100.0$ \\
\hline Coughing & $29.3(25.4)$ & 33.3 & $0.0-100.0$ \\
\hline Feeling ill & $29.3(25.3)$ & 33.3 & $0.0-100.0$ \\
\hline
\end{tabular}

Abbreviations: EORTC QLQ-C30: European Organization for Research and Treatment of Cancer Quality of Life Questionnaire C30; H\&N35: Head and Neck Module; SD: standard deviation.

$0.034)$, tumor sits at nasopharynx $(\beta=-9.1, \mathrm{p}=0.034)$, or treated by 2DRT $(\beta=-12.2, \mathrm{p}<0.01)$ had a higher probability to report a high level of xerostomia. Meanwhile, survivors with family annual income $\geqq 1.2$ million NTD $(\beta=5.2, \mathrm{p}<0.01), \mathrm{CCI}=0(\beta=-6.1, \mathrm{p}<0.01)$, or treated by 3DCRTor IMRT $(\beta=7.7, \mathrm{p}<0.01)$ had a higher probability of reporting a better global QoL.

\section{Tumor site and HR-QoL outcome}

Significant difference $(p<0.05)$ of HR-QoL outcome at different tumor sites was observed at 11 of the 15 scales in QLQ-C30 and 10 of the 13 scales in H\&N35 (Table 5 ). Compared with nasopharyngeal cancer survivors, three worse scales (social eating, social contact, and opening mouth) were significantly observed on oral cancer survivors, so were six scales (appetite loss, pain, swallowing, speech, social eating, and social contact) on oropharyngeal cancer survivors and eight scales (physical functioning, role functioning, dyspnea, constipation, swallowing, speech, social contact, coughing) on hypopharyneal/laryngeal cancer survivors. Meanwhile, compared with oral cavity survivors, five worse scales (cognitive functioning, fatigue, nausea/vomiting, appetite loss, and pain) were observed in oropharyngeal cancer survivors, and five worse scales (physical functioning, nausea/vomiting, dyspnea, speech, and coughing) in hypopharyneal/laryngeal cancer survivors. On the contrast, better results in opening mouth and dry mouth were observed in hypopharyneal/laryngeal cancer survivors compared with the other survivors.

\section{RT techniques and HR-QoL outcome}

Significant difference $(p<0.05)$ of HR-QoL outcome by different RT techniques was observed at 2 of the 15 scales in QLQ-C30 and 10 of the 13 scales in H\&N35 (Table 6). Compared with 2DRT, IMRT had significant better outcome in the scales of global QoL, physical functioning, swallowing, senses (taste/smell), speech, social eating, social contact, teeth, opening mouth, dry mouth, sticky saliva, and feeling ill. Only three scales (teeth, dry mouth, and sticky saliva) with better results were observed in 3DCRT compared with 2DRT. IMRT had better scores in most scales compared with 3DCRT, but without reaching statistically significant difference.

\section{Discussion}

The treatment fields of conventional 2DRT for HNC are usually large and anatomic structures situated nearby the tumors, such as the salivary glands, inner ear, oral cavity, and temporomandibular joints, are exposed to almost the same irradiated dose as the treatment target sites. We have previously used the Taiwan Chinese version of SF-36 to investigate the HR-QoL of oral cancer or nasopharyngeal survivors treated by conventional RT, finding that these HNC survivors scored significantly worse in most of the eight functional domains of SF-36 than Taiwanese norms $[15,16]$. However, SF-36 is not specific and sensitive enough as a HR-QoL instrument to discriminate the effect differences for head- and neck-related problems between different treatment modalities. In the present study, we used the EORTC QLQC30 and H\&N35 to assess HR-QoL for HNC patients. The Taiwan Chinese version of the questionnaires has been previously tested in HNC patients by Chie et al. [23]. They found the Cronbach's alpha coefficients of all scales of the two questionnaires were $\geqq 0.70$, except that for cognitive functioning, and the correlation of scales that measure similar dimensions of the QLQ-C30 and the SF-36 was moderate to high, whereas that of the H\&N35 and the QLQ-C30 or the SF-36 was moderate to low. 
Table 3 GLM-MANOVA test of the overall effect of the sociodemographic and clinical variables on the EORTC QLQ-C30 and H\&N35 scales

\begin{tabular}{|c|c|c|c|c|c|c|c|c|}
\hline \multirow[b]{3}{*}{ Variable } & \multicolumn{4}{|l|}{ QLQ-C30 } & \multicolumn{4}{|c|}{ QLQ-H\&N35 } \\
\hline & \multicolumn{2}{|c|}{$\begin{array}{l}\text { One-factor } \\
\text { model* }\end{array}$} & \multicolumn{2}{|c|}{$\begin{array}{l}\text { Multifactor } \\
\text { model }^{* *}\end{array}$} & \multicolumn{2}{|c|}{$\begin{array}{l}\text { One-factor } \\
\text { model }^{*}\end{array}$} & \multicolumn{2}{|c|}{$\begin{array}{l}\text { Multifactor } \\
\text { model }^{* *}\end{array}$} \\
\hline & Wilk's $\lambda$ & $p$ & Wilk's $\lambda$ & $p$ & Wilk's $\lambda$ & $p$ & Wilk's $\lambda$ & $p$ \\
\hline \multicolumn{9}{|l|}{ Sociodemographic variables } \\
\hline Age: $<40 \vee 40 \sim 60 \vee \geq 60$ years & 0.922 & 0.016 & 0.942 & NS & 0.926 & 0.013 & 0.939 & NS \\
\hline Gender: female $v$ male & 0.890 & $<0.01$ & 0.877 & $<0.01$ & 0.919 & 0.018 & 0.963 & NS \\
\hline Education years: $\leq 6 \vee 6 \sim 12 \vee>12$ & 0.914 & 0.011 & 0.928 & NS & 0.920 & 0.033 & 0.941 & NS \\
\hline Marital status: with $\vee$ without spouse & 0.958 & 0.042 & 0.951 & NS & 0.975 & NS & 0.968 & NS \\
\hline Annual family income: $<0.6 \vee 0.6 \sim 1.2 \vee \geq 1.2$ (million NTD) & 0.845 & $<0.01$ & 0.896 & 0.032 & 0.866 & $<0.01$ & 0.910 & NS \\
\hline \multicolumn{9}{|l|}{ Clinical variables } \\
\hline CCl score: $0 \vee \geq 1$ & 0.937 & 0.001 & 0.910 & NS & 0.965 & NS & 0.935 & NS \\
\hline Tumor site: Oral cavity v Oropharynx v Hypopharynx/Larynx v Nasopharynx & 0.805 & $<0.01$ & 0.828 & $<0.01$ & 0.618 & $<0.01$ & 0.824 & $<0.01$ \\
\hline AJCC stage: stage I-II $\vee \| I-I V$ & 0.870 & $<0.01$ & 0.863 & $<0.01$ & 0.880 & $<0.01$ & 0.869 & NS \\
\hline Treatment methods: $S+R T \vee S+R T+C / T \vee R T \pm C / T$ & 0.865 & $<0.01$ & 0.845 & $<0.01$ & 0.832 & $<0.01$ & 0.906 & NS \\
\hline RT technique: 2DRT $\vee 3 D C R T \vee$ IMRT & 0.888 & $<0.01$ & 0.876 & $<0.01$ & 0.869 & $<0.01$ & 0.829 & $<0.01$ \\
\hline Follow-up years: $2 \sim 3 \vee 3 \sim 5 \vee>5$ & 0.937 & NS & 0.928 & NS & 0.933 & NS & 0.938 & NS \\
\hline
\end{tabular}

*: The one factor model: only one independent variable was entered into the model. **: The multifactor model: all mentioned variables were entered as independent variables in the model. Abbreviations: GLM-MANOVA: general linear model multivariate of variance; EORTC QLQ-C30: European Organization for Research and Treatment of Cancer Quality of Life Core questionnaire; H\&N35: Head and Neck Module; NTD: New Taiwan Dollar (1 USD = 33 NTD); CCl: Charlson comorbidity index; AJCC: American Joint of Cancer Committee published in 2002; S: surgery; RT: radiotherapy; C/T: chemotherapy; 2DRT: two dimensional RT; 3DCRT: three dimensional conformal RT; IMRT: intensity modulated RT; NS: not significant.

Growing reports have shown that the dosimetric superiority of 3DCRT/IMRT applied in HNC patients can not only preserve salivary functions, but also improve local control, and even patient survival [10-12]. The current study presented with a large cohort to compare HR-QoL between HNC survivors receiving different RT techniques applied at a single institute during different time periods, adjusting for major sociodemographic and medical variables that affect these measures. Although heterogeneity existed in the comparing groups, the potential bias of patient selection was reduced, and the contributions of the different RT techniques in producing particular HR-QoL outcomes were highlighted. We demonstrated that the use of IMRT significantly improved HR-QoL for HNC survivors as compared with those treated by conventional 2DRT.

Xerostomia related symptoms were usually cited as the most prevalent complications in HNC survivors post $\mathrm{RT}$ and patient-reported xerostomia has been found to significantly correlate with mean dose to the parotid

Table 4 Multiple linear regression analysis for global quality of life and xerostomia

\begin{tabular}{|c|c|c|c|c|c|c|}
\hline \multirow[b]{2}{*}{ Variable } & \multicolumn{3}{|c|}{ Xerostomia } & \multicolumn{3}{|c|}{ Global quality of life } \\
\hline & $\beta$ & SE & $\mathrm{p}$ & $\beta$ & SE & $\mathrm{p}$ \\
\hline \multicolumn{7}{|l|}{ Sociodemographic variables } \\
\hline Age: $<40 \vee 40 \sim 60 \vee \geq 60$ years & -0.7 & 2.5 & NS & -0.2 & 1.6 & NS \\
\hline Gender: female $v$ male & -1.7 & 3.5 & NS & 0.5 & 2.2 & NS \\
\hline Education years: $\leq 6 \vee 6 \sim 12 \vee>12$ & 1.2 & 3.6 & NS & 3.2 & 1.3 & NS \\
\hline Marital status: with $\vee$ without spouse & -2.6 & 3.1 & NS & -3.0 & 2.0 & NS \\
\hline Annual family income: $<1.2 \vee \geq 1.2$ (million NTD) & -4.0 & 2.5 & NS & 5.2 & 1.6 & $<0.01$ \\
\hline \multicolumn{7}{|l|}{ Clinical variables } \\
\hline CCl score: $0 \vee \geq 1$ & 5.2 & 2.5 & 0.034 & -6.1 & 1.6 & $<0.01$ \\
\hline Tumor site: Nasopharynx $v$ others & -9.1 & 4.3 & 0.036 & -0.9 & 2.7 & NS \\
\hline AJCC stage: stage I-II $\vee \| I-I V$ & 1.5 & 3.1 & NS & 0.2 & 2.0 & NS \\
\hline Treatment methods: $S+R T \vee S+R T+C / T \vee R T \pm C / T$ & 3.0 & 3.7 & NS & -1.8 & 2.3 & NS \\
\hline RT technique: 2DRT $\vee$ 3DCRT/IMRT & -12.2 & 2.7 & $<0.01$ & 7.7 & 1.7 & $<0.01$ \\
\hline Follow-up years: $2 \sim 3 \vee 3 \sim 5 \vee>5$ & 0.2 & 2.9 & NS & 1.6 & 1.9 & NS \\
\hline
\end{tabular}

$\beta$ : un-standardized regression coefficient; SE: standard error; R2 $=0.072$ in the model for xerostomia; R2 $=0.077$ in the model for global quality of life; NTD: New Taiwan Dollar (1 USD = 33 NTD); CCI: Charlson comorbidity index; AJCC: American Joint of Cancer Committee published in 2002; S: surgery; RT: radiotherapy; C/T: chemotherapy; 2DRT: two dimensional RT; 3DCRT: three dimensional conformal RT; IMRT: intensity modulated RT; NS: not significant. 
Table 5 The comparisons of EORTC QLQ-C30 and H\&N35 scales for head and neck cancer survivors at different tumor sites

\begin{tabular}{|c|c|c|c|c|c|}
\hline & $\begin{array}{l}\text { Nasopharynx } \\
(\mathrm{n}=316)\end{array}$ & $\begin{array}{l}\text { Oral cavity } \\
(\mathrm{n}=129)\end{array}$ & $\begin{array}{l}\text { Oropharynx } \\
(n=75)\end{array}$ & $\begin{array}{l}\text { Hypropharynx } \\
\text { \&Larynx }(n=120)\end{array}$ & \\
\hline & Mean (SD) & Mean (SD) & Mean (SD) & Mean (SD) & $p$ \\
\hline \multicolumn{6}{|l|}{ EORTC QLQ-C30 } \\
\hline Global quality of life & $53.7(20.4)$ & $56.1(16.7)$ & $55.2(21.6)$ & $54.8(20.9)$ & NS \\
\hline Physical functioning & $85.7(16.3)$ & 86.4 (13.8)e & $82.2(16.9)$ & $80.9(18.7) \mathrm{c}$ & 0.015 \\
\hline Role functioning & $88.7(19.5)$ & $87.0(19.1)$ & $83.6(22.1)$ & $81.2(25.0) \mathrm{C}$ & $<0.01$ \\
\hline Emotional functioning & $78.0(19.7)$ & 79.9 (18.6) & $75.3(22.7)$ & $77.0(20.8)$ & NS \\
\hline Cognitive functioning & $77.8(18.7)$ & $82.8(19.2) d$ & $75.1(23.0)$ & $76.9(20.9)$ & 0.025 \\
\hline Social functioning & $77.0(23.2)$ & $75.4(22.8)$ & $73.1(27.0)$ & $71.0(25.8)$ & NS \\
\hline Fatigue & $29.6(19.8)$ & $24.9(21.2) d$ & $32.7(19.2)$ & $30.4(21.4)$ & 0.037 \\
\hline Nausea/Nomiting & $8.4(15.6)$ & $6.2(14.2) d, e$ & $13.5(22.5)$ & $12.4(20.8)$ & $<0.01$ \\
\hline Pain & $20.8(21.5)$ & $19.2(23.1)$ & $26.8(21.9)$ & $24.9(23.8)$ & 0.038 \\
\hline Dyspnea & $12.7(19.9)$ & $12.4(20.4) \mathrm{e}$ & $17.8(23.5)$ & $23.6(24.0) c$ & $<0.01$ \\
\hline Insomnia & $25.1(26.3)$ & $23.1(25.5)$ & $30.1(22.8)$ & $30.7(26.5)$ & 0.049 \\
\hline Appetite loss & $17.0(23.8)$ & $17.9(24.3) d$ & $30.7(26.0) b$ & $23.1(24.1)$ & $<0.01$ \\
\hline Constipation & $16.5(22.4)$ & $18.4(22.4)$ & $23.7(26.8)$ & $24.0(25.3) \mathrm{c}$ & $<0.01$ \\
\hline Diarrhea & $13.4(18.9)$ & $12.2(18.6)$ & $16.4(26.0)$ & $15.9(19.3)$ & NS \\
\hline Financial problems & $23.4(26.4)$ & $26.9(25.7)$ & $31.9(29.9)$ & $30.5(27.9)$ & 0.023 \\
\hline \multicolumn{6}{|l|}{ EORTC QLQ-HN35 } \\
\hline Pain & $17.0(18.3)$ & $17.3(22.9) \mathrm{d}$ & $27.4(20.7) b$ & $21.5(22.5)$ & $<0.01$ \\
\hline Swallowing & $26.8(22.4)$ & $30.6(25.4)$ & $38.0(23.6) \mathrm{b}$ & $33.8(27.5) \mathrm{c}$ & $<0.01$ \\
\hline Senses (taste/smell) & $27.2(27.0)$ & $22.1(26.8)$ & $26.4(27.4)$ & $31.3(30.1)$ & NS \\
\hline Speech & $23.0(23.3)$ & $25.6(21.8) \mathrm{e}$ & $32.0(27.1) \mathrm{b}$ & $41.3(30.7) c$ & $<0.01$ \\
\hline Social eating & $24.1(24.9)$ & $33.2(27.0) a$ & $35.4(27.8) b$ & $30.7(29.5)$ & $<0.01$ \\
\hline Social contact & $15.4(19.9)$ & $24.0(23.2) a$ & $23.9(26.7) \mathrm{b}$ & $27.5(27.4) c$ & $<0.01$ \\
\hline Sexuality & $23.8(27.6)$ & $24.3(24.4)$ & $30.5(29.4)$ & $31.3(29.5)$ & 0.035 \\
\hline Teeth & $38.1(28.3)$ & $41.0(31.8)$ & $42.7(30.2)$ & $36.1(31.3)$ & NS \\
\hline Opening mouth & $30.1(29.7)$ & 43.9 (34.2)a, e & $37.8(32.9) f$ & $25.1(29.4)$ & $<0.01$ \\
\hline Dry mouth & $53.5(31.3)$ & $45.9(30.7)$ & $49.2(30.6) f$ & $36.6(27.5) c$ & $<0.01$ \\
\hline Sticky saliva & $42.8(31.7)$ & $37.6(28.4)$ & $46.3(29.8)$ & $35.0(29.8)$ & 0.024 \\
\hline Coughing & $25.9(23.9)$ & 23.9 (23.3)e & $31.5(27.4) f$ & $43.1(25.4) \mathrm{c}$ & $<0.01$ \\
\hline Feeling ill & $29.6(25.5)$ & $24.8(23.7)$ & $31.5(25.1)$ & $32.1(26.0)$ & NS \\
\hline
\end{tabular}

$\mathrm{a}: \mathrm{p}<0.05$, Oral cavity compared with Nasopharynx; b : p < 0.05, Oropharynx compared with Nasopharynx; $: \mathrm{p}<0.05, \mathrm{Hypropharynx} \mathrm{\&} \mathrm{Larynx} \mathrm{compared} \mathrm{with}$ Nasopharynx; $d: p<0.05$, Oral cavity compared with Oropharynx; e : p $<0.05$, Oral cavity compared with Hypropharynx \& Larynx; $: p<0.05$, Oropharynx compared with Hypropharynx \& Larynx.

glands and the minor salivary glands in the oral cavity [26]. In a matched comparison of 67 pairs of HNC survivors treated by 2DRT versus IMRT, Graff et al. observed the major advantages of IMRT were on oral symptoms, especially salivary dysfunction and oral discomfort [27]. Similarly, a cross sectional survey of 163 HNC survivors by van Rij et al. also revealed that parotid sparing IMRT for HNC patients improved xerostomia related HR-QoL compared to 2DRT both in rest and during meals [28]. With the large cohort of 640 HNC survivors in current study, we confirmed that RT technique is the determining variable affecting xerostomia in HNC survivors and affirmed that modern RT, especially for IMRT, not only reduced the oral related symptoms but also the global QoL.
However, the direct cause-effect relationship between xerostomia and broad aspect of HR-QoL in HNC patients after RT has not been established yet. The improvement of IMRT in HR-QoL might not be only through reducing xerostomia by salivary glands sparing, but also reducing the volume of other non-target organs receiving a high dose. Some reports have shown that radiation-induced dysphagia also plays an important role in HR-QoL domains [28-30]. Dysphagia is usually multifactorial and strongly associated with xerostomia. It has become increasingly important to identify the anatomical structures that are involved in swallowing problems after RT. A cross-sectional study of 81 patients with oropharyngeal cancer, reported by Levendag et al., showed that the probability of swallowing complaints is 
Table 6 The comparisons of EORTC QLQ-C30 and H\&N35 scales for head and neck cancer survivors by different RT techniques

\begin{tabular}{|c|c|c|c|c|}
\hline & $\begin{array}{l}\text { 2DRT } \\
(n=371)\end{array}$ & $\begin{array}{l}\text { 3DCRT } \\
(n=127)\end{array}$ & $\begin{array}{l}\text { IMRT } \\
(n=142)\end{array}$ & \\
\hline & Mean (SD) & Mean (SD) & Mean (SD) & $p$ \\
\hline \multicolumn{5}{|l|}{ EORTC QLQ-C30 } \\
\hline Global quality of life & $51.7(19.9)$ & $56.2(18.6)$ & $60.7(19.7) b$ & $<0.01$ \\
\hline Physical functioning & $83.1(17.7)$ & $86.2(14.1)$ & $86.7(14.6) b$ & 0.039 \\
\hline Role functioning & $85.6(22.2)$ & $86.7(19.4)$ & $88.1(19.1)$ & NS \\
\hline Emotional functioning & $76.6(20.8)$ & $79.8(18.0)$ & $79.6(19.8)$ & NS \\
\hline Cognitive functioning & $77.2(20.7)$ & $79.9(16.6)$ & $80.0(20.1)$ & NS \\
\hline Social functioning & $73.4(26.0)$ & $76.7(20.3)$ & $78.1(22.0)$ & NS \\
\hline Fatigue & $31.0(21.1)$ & $26.9(16.8)$ & $26.5(21.0)$ & NS \\
\hline NauseaNomiting & $10.3(19.2)$ & $7.7(13.6)$ & $8.2(15.5)$ & NS \\
\hline Pain & $23.9(24.1)$ & $18.5(16.9)$ & $19.7(21.7)$ & NS \\
\hline Dyspnea & $16.2(23.4)$ & $15.8(18.8)$ & $12.1(18.7)$ & NS \\
\hline Insomnia & $26.9(27.5)$ & $25.6(22.1)$ & $25.4(25.0)$ & NS \\
\hline Appetite loss & $21.7(26.7)$ & $18.8(20.9)$ & $16.4(21.2)$ & NS \\
\hline Constipation & $19.8(25.2)$ & $19.2(22.5)$ & $17.1(20.4)$ & NS \\
\hline Diarrhea & $14.8(21.3)$ & $12.4(17.8)$ & $13.3(17.6)$ & NS \\
\hline Financial problems & $27.1(28.1)$ & $23.1(24.1)$ & $27.6(26.7)$ & NS \\
\hline \multicolumn{5}{|l|}{ EORTC QLQ-HN35 } \\
\hline Pain & $20.8(22.6)$ & $18.4(16.7)$ & $15.2(17.7)$ & NS \\
\hline Swallowing & $33.3(25.6)$ & $28.0(22.4)$ & $23.9(21.5) b$ & $<0.01$ \\
\hline Senses (taste/smell) & $29.4(29.0)$ & $26.3(28.1)$ & $20.6(22.7) b$ & $<0.01$ \\
\hline Speech & $29.6(26.4)$ & $29.5(25.7)$ & $22.4(24.0) b$ & 0.013 \\
\hline Social eating & $31.0(27.5)$ & $28.5(27.0)$ & $21.9(24.3) \mathrm{b}$ & $<0.01$ \\
\hline Social contact & $21.0(24.1)$ & $22.7(24.7)$ & $16.6(20.3) b$ & $<0.01$ \\
\hline Sexuality & $27.4(28.9)$ & $27.0(28.0)$ & $21.5(23.8)$ & NS \\
\hline Teeth & $43.2(30.6)$ & 34.7 (29.1)a & $31.2(26.0) b$ & $<0.01$ \\
\hline Opening mouth & $36.5(31.7)$ & $30.2(30.4)$ & $25.4(31.1) \mathrm{b}$ & $<0.01$ \\
\hline Dry mouth & $54.0(31.9)$ & $43.8(28.2) \mathrm{a}$ & $41.0(27.9) \mathrm{b}$ & $<0.01$ \\
\hline Sticky saliva & $45.1(32.3)$ & 35.8 (26.1)a & $33.6(27.9) \mathrm{b}$ & $<0.01$ \\
\hline Coughing & $31.0(26.9)$ & $29.5(24.2)$ & $24.5(21.7)$ & NS \\
\hline Feeling ill & $32.3(26.3)$ & $26.3(23.0)$ & $24.0(23.2) b$ & $<0.01$ \\
\hline
\end{tabular}

Abbreviations: RT = radiotherapy; 2DRT = two dimensional RT; 3DCRT = three dimensional conformal RT; IMRT = intensity-modulated RT; a: $\mathrm{p}<0.05$, 3DCRT compared with 2DRT; b: $p<0.05$, IMRT compared with 2DRT

significantly associated with the mean total radiation dose in the superior and middle pharyngeal constrictor muscle [29]. Our data confirmed this hypothesis that the use of 3DCRT/IMRT also reduced other head-andneck-related symptom scales to some extent. The interand intra-scale correlations of EORTC QLQ-C30 and QLQ-H\&N35 were significantly high in our series [data not shown], which means the symptomatic problems improved by 3DCRT/IMRT are inter-correlative and might converge and reflect to the expression of patients' global QoL.

The comparisons between 3DCRT and IMRT were mainly on the dosimetric distribution in the literature, and the information concerning the clinical advantage attributed to the evolution from 3DCRT to IMRT in HNC patients is still limited and controversial. In our previous study exclusively focusing on nasopharyngeal cancer, a significant reduction of $25-30 \%$ of the mean dose to the normal structures such as parotid glands and oral cavity was created by IMRT compared with 3DCRT. However, comparing their longitudinal changes of HR-QoL, we did not find a significant difference at most time points except 3 months after RT [31]. Current study echoes this finding that the positive advantage of IMRT over 3DCRT in QoL is ambiguous and marginal in HNC survivors. In contrast, a non-randomized prospective comparison between HNC patients treated by 3DCRT or IMRT demonstrated that IMRT resulted in a significant reduction of patient- and observer-rated xerostomia, as well as other head and neck symptoms, compared with standard 3DCRT [32]. With locally advanced disease treated by aggressive combined 
modality in most of our subjects, we found that the dosimetric improvement for 3DCRT compared with IMRT might not be sufficiently large to demonstrate any significant difference in HR-QoL.

Besides radiation technique, socioeconomic status, comorbidity, and tumor site were also found to be significant prognosticators on HR-QoL outcome. With the relatively heterogeneous nature of $\mathrm{HNC}$ patients and experienced treatments including varying tumor stages, sites, and the frequently diverse treatment modalities applied and involved administering institutions, the factors affecting the HR-QoL after treatment for HNC patients usually appear to be somewhat discordant and complicated in the literature. For example, Hammerlid et al. found those HNC survivors with tumor located at larynx, aged below 65 years, or female patients had significantly better HR-QoL than their counterparts three years after treatment [33] and de Graeff et al. reported female sex, higher cancer stage, and combination treatment were found to be associated with more symptomatic problems and worse HR-QoL [34].

Although being comprehensive and well validated with recognized internal consistency and reliability, EORTC QLQ-C30 and QLQ-H\&N35 still have some limitations in the interpretation of HR-QoL of HNC patients, because they do not deal with some specific but common late sequelae, such as deafness, otitis media, symptoms from temporal lobe necrosis, or radiation neuropathy, or hypopituitarism in nasopharyngeal cancer survivors. A tumor site-specific assessment tool of HR-QoL might provide more specific and sensitive information to discriminate site-related differences of HR-QoL in HNC patients with various tumor sites treated by different strategies. Furthermore, without pre-treatment HR-QoL data available in our cohort, potential selection bias might still exist in the cross sectional study, though the confounding variables adjusted by multivariate analysis. A longitudinal study assessing the changes of HR-QoL will be justified to more accurately detect the differences between the groups.

\section{Conclusions}

With the advance of modern RT technology, head-andneck related symptoms after RT could be significantly reduced and reflected to the improvement of broad aspects of HR-QoL in HNC survivors. However, there may still be some undetected factors, which are related to global QoL or some specific functional domains, to be explored in future investigation.

\section{Abbreviations}

HR-QoL: health-related quality of life; HNC: head and neck cancer; EORTC QLQ-C30: European Organization for Research and Treatment of Cancer
Quality of Life Core questionnaire; H\&N35: Head and Neck Module; GLMMANOVA: general linear model multivariate of variance; NTD: New Taiwan Dollar (1 USD = 33 NTD); CCl: Charlson comorbidity index; AJCC: American Joint of Cancer Committee published in 2002; S: surgery; RT: radiotherapy; C/ $\mathrm{T}$ : chemotherapy; 2DRT: two dimensional RT; 3DCRT: three dimensional conformal RT; IMRT: intensity modulated RT; $\beta$ : un-standardized regression coefficient; SE: standard error; NS: not significant.

\section{Acknowledgements}

The study was supported by the grants "CMRPG860501", "CMRPG860502", "CMRPG890062", and "NSC 99-2221-E-151-010".

\section{Author details}

${ }^{1}$ Department of Radiation Oncology, Yuan's General Hospital, Kaohsiung City, Taiwan. ${ }^{2}$ Department of Radiological Technology, Central Taiwan University of Science and Technology, Taichung City, Taiwan. ${ }^{3}$ Medical Physics \& Informatics Lab., Department of Electronics Engineering, National Kaohsiung University of Applied Sciences, Kaohsiung, Taiwan. ${ }^{4}$ Department of Otolaryngology, Chang Gung Memorial Hospital - Kaohsiung Medical Center, Chang Gung University College of Medicine, Kaohsiung, Taiwan. ${ }^{5}$ Radiation Oncology, Chang Gung Memorial Hospital - Kaohsiung Medical Center, Chang Gung University College of Medicine, Kaohsiung, Taiwan. ${ }^{6}$ Department of Biotechnical Cosmetology, Cheng Shiu University, Kaohsiung, Taiwan.

\section{Authors' contributions}

SW Leung and TFL: writing of manuscript and study coordinator. FMF: original idea, concept and final revision of manuscript. CC, TFL, WLT and PJC: design and development of study. WLT: statistical analysis. All authors read and approved the final manuscript

\section{Competing interests}

The authors declare that they have no competing interests.

Received: 12 August 2010 Accepted: 12 April 2011

Published: 12 April 2011

\section{References}

1. List M, Stracks J: Evaluation of quality of life in patients definitively treated for squamous carcinoma of the head and neck. Curr Opin Oncol 2000, 12:215.

2. Ware J, Kosinski M, Keller S: SF-36 physical and mental health summary scales: a user's manual. 1994.

3. Ware J Jr, Sherbourne C: The MOS 36-item short-form health survey (SF36): I. Conceptual framework and item selection. Med Care 1992, 30:473-483.

4. Aaronson N, Ahmedzai S, Bergman B, et al: The European Organization for Research and Treatment of Cancer QLQ-C30: a quality-of-life instrument for use in international clinical trials in oncology. J Natl Cancer Inst 1993, 85:365.

5. Cella D, Tulsky D, Gray G, et al: The Functional Assessment of Cancer Therapy scale: development and validation of the general measure. J Clin Oncol 1993, 11:570.

6. Bjordal K, Hammerlid E, Ahlner-Elmqvist M, et al: Quality of life in head and neck cancer patients: validation of the European Organization for Research and Treatment of Cancer Quality of Life Questionnaire-H\&N35. J Clin Oncol 1999, 17:1008.

7. Amdur R, Parsons J, Mendenhall W, et al: Postoperative irradiation for squamous cell carcinoma of the head and neck: an analysis of treatment results and complications. Int J Radiat Oncol Biol Phys 1989, 16:25-36.

8. Teo P, Ma B, Chan A: Radiotherapy for nasopharyngeal carcinomatransition from two-dimensional to three-dimensional methods. Radiother Oncol 2004, 73:163-172.

9. Chao K, Wippold F: Determination and delineation of nodal target volumes for head-and-neck cancer based on patterns of failure in patients receiving definitive and postoperative IMRT* 1 . Int J Radiat Oncol Biol Phys 2002, 53:1174-1184.

10. Saarilahti K, Kouri M, Collan J, et al: Intensity modulated radiotherapy for head and neck cancer: evidence for preserved salivary gland function. Radiother Oncol 2005, 74:251-258. 
11. Kwong D, Pow E, Sham J, et al: Intensity-modulated radiotherapy for early-stage nasopharyngeal carcinoma. Cancer 2004, 101:1584-1593.

12. Sultanem K, Shu H, Xia P, et al: Three-dimensional intensity-modulated radiotherapy in the treatment of nasopharyngeal carcinoma: the University of California-San Francisco experience. Int J Radiat Oncol Biol Phys 2000, 48:711-722.

13. Lee N, Xia P, Quivey J, et al: Intensity-modulated radiotherapy in the treatment of nasopharyngeal carcinoma: an update of the UCSF experience. Int J Radiat Oncol Biol Phys 2002, 53:12-22.

14. Wolden S, Chen W, Pfister D, et al: Intensity-modulated radiation therapy (IMRT) for nasopharynx cancer: update of the Memorial Sloan-Kettering experience. Int J Radiat Oncol Biol Phys 2006, 64:57-62.

15. Fang F, Tsai W, Chien C, Chiu H, Wang C: Health-related quality of life outcome for oral cancer survivors after surgery and postoperative radiotherapy. Jpn J Clin Oncol 2004, 34:641.

16. Fang $\mathrm{F}, \mathrm{Chiu} \mathrm{H}, \mathrm{Kuo} W$, et al: Health-related quality of life for nasopharyngeal carcinoma patients with cancer-free survival after treatment. Int J Radiat Oncol Biol Phys 2002, 53:959-968.

17. Charlson M, Pompei $P$, Ales $K$, Mackenzie C: A new method of classifying prognostic comorbidity in longitudinal studies: Development and validation. J Chro Diseas 1987, 40:373-383

18. Fang F, Leung S, Huang C, et al: Combined-modality therapy for squamous carcinoma of the buccal mucosa: treatment results and prognostic factors. Head Neck 1997, 19:506-512.

19. Fang F, Leung S, Wang C, et al: Computed tomography findings of bony regeneration after radiotherapy for nasopharyngeal carcinoma with skull base destruction: implications for local control. Int J Radiat Oncol Biol Phys 1999, 44:305-309.

20. Hsiung $C$, Yorke $E_{\text {, Chui }}$, et al: Intensity-modulated radiotherapy versus conventional three-dimensional conformal radiotherapy for boost or salvage treatment of nasopharyngeal carcinoma. Int J Radiat Oncol Biol Phys 2002, 53:638-647.

21. Hsiung $C$, Hunt $M$, Yorke $E$, et al: Intensity-modulated radiotherapy as the boost or salvage treatment of nasopharyngeal carcinoma: The appropriate parameters in the inverse planning and the effect of patient's anatomic factors on the planning results. Radiother Oncol 2005, 77:53-57.

22. Bjordal K, Ahlner-Elmqvist M, Hammerlid E, et al: A prospective study of quality of life in head and neck cancer patients. Part II: Longitudinal data. Laryngoscope 2001, 111:1440-1452.

23. Chie W, Hong R, Lai C, Ting L, Hsu M: Quality of life in patients of nasopharyngeal carcinoma: validation of the Taiwan Chinese version of the EORTC QLQ-C30 and the EORTC QLQ-H\&N35. Qual Life Res 2003, 12:93-98.

24. Fayers P, Bottomley A: Quality of life research within the EORTC-the EORTC QLQ-C30. Eur J Cancer 2002, 38:125-133.

25. Middel B, Stewart R, Bouma J, Sonderen E, Heuvel W: How to validate clinically important change in health related functional status. Is the magnitude of the effect size consistently related to magnitude of change as indicated by a global question rating? J Eval Clin Pract 2001, 7:399-410.

26. Eisbruch A, Kim H, Terrell J, et al: Xerostomia and its predictors following parotid-sparing irradiation of head-and-neck cancer. Int J Radiat Oncol Biol Phys 2001, 50:695-704

27. Graff $P$, Lapeyre M, Desandes $E$, et al: Impact of intensity-modulated radiotherapy on health-related quality of life for head and neck cancer patients: matched-pair comparison with conventional radiotherapy. Int J Radiat Oncol Biol Phys 2007, 67:1309-1317.

28. van Rij C, Oughlane-Heemsbergen W, Ackerstaff A, et al: Parotid gland sparing IMRT for head and neck cancer improves xerostomia related quality of life. Radiat Oncol 2008, 3:41.

29. Levendag P, Teguh D, Voet P, Van der E: Dysphagia disorders in patients with cancer of the oropharynx are significantly affected by the radiation therapy dose to the superior and middle constrictor muscle: a doseeffect relationship. Radiother Oncol 2007, 85:64-73.

30. Teguh D, Levendag P, Noever I, et al: Treatment techniques and site considerations regarding dysphagia-related quality of life in cancer of the oropharynx and nasopharynx. Int J Radiat Oncol Biol Phys 2008, 72:1119-1127.

31. Fang F, Chien C, Tsai W, et al: Quality of life and survival outcome for patients with nasopharyngeal carcinoma receiving three-dimensional conformal radiotherapy vs. intensity-modulated radiotherapy - a longitudinal study. Int J Radiat Oncol Biol Phys 2008, 72:356-364.

32. Vergeer $M$, Doornaert $P$, Rietveld $D$, et al: Intensity-modulated radiotherapy reduces radiation-induced morbidity and improves healthrelated quality of life: results of a nonrandomized prospective study using a standardized follow-up program. Int J Radiat Oncol Biol Phys 2009, 74:1-8.

33. Hammerlid E, Taft C: Health-related quality of life in long-term head and neck cancer survivors: a comparison with general population norms. Brit J cancer 2001, 84:149.

34. Alexander de Graeff J, de Leeuw W, Gert-Jan Hordijk G, Jacques A: Longterm quality of life of patients with head and neck cancer. Laryngoscope 2000, 110:98-106.

\section{Pre-publication history}

The pre-publication history for this paper can be accessed here: http://www.biomedcentral.com/1471-2407/11/128/prepub

doi:10.1186/1471-2407-11-128

Cite this article as: Wan Leung et al:: Health-related Quality of life in 640 head and neck cancer survivors after radiotherapy using EORTC QLQ-C30 and QLQ-H\&N35 questionnaires. BMC Cancer 2011 11:128.

\section{Submit your next manuscript to BioMed Central and take full advantage of:}

- Convenient online submission

- Thorough peer review

- No space constraints or color figure charges

- Immediate publication on acceptance

- Inclusion in PubMed, CAS, Scopus and Google Scholar

- Research which is freely available for redistribution

Submit your manuscript at www.biomedcentral.com/submit
Ciomed Central 